\title{
Rigid Isotopy Classification of Real Algebraic Curves of Bidegree $(3,3)$ on Quadrics
}

\section{A.I.Degtyarev, V.I.Zvonilov}

\begin{abstract}
A rigid isotopy of nonsingular real algebraic curves on a quadric is a path in the space of such curves of a given bidegree. We obtain the rigid isotopy classification of nonsingular real algebraic curves of bidegree $(3,3)$ on a hyperboloid and on an ellipsoid. We also study the space of real algebraic curves of bidegree $(3,3)$ with a single node or cusp. Bibliography: 11 items.
\end{abstract}

1. Introduction. The notion of rigid isotopy was introduced by Rokhlin [1]. On the projective plane the classification of nonsingular real algebraic curves up to rigid isotopy is known for degree $m \leq 6$ (see [1], [2], [3]). On quadrics rigid isotopies of real algebraic curves of bidegrees $(m, 1),(m, 2)$ were studied by the authors, see [4], [5], where the rigid isotopy classification of such nonsingular curves is obtained. For (nonsingular) curves of bidegree $(3,3)$ on quadrics the classification of their real schemes (i.e., real isotopy classification) was obtained in [6] and [7] (see also [8]), and the classification of their complex schemes (i.e., real schemes enriched with a type and complex orientations, see below), in [8]. In the present paper we prove that a nonsingular curve of bidegree $(3,3)$ on a hyperboloid (on an ellipsoid) is determined up to rigid isotopy by its complex (respectively, real) scheme, see Theorem 2. In the proof of Theorem 1 we enumerate all the connected components of the space of curves of bidegree $(3,3)$ with a single node or cusp (see Figures 1 and 2). We use the approach to the rigid isotopy classification of plane real quartics suggested in [9].

2. Definitions and notation. Let $X$ be a nonsingular quadric. The complex part $C X$ of $X$ is $C P^{1} \times C P^{1}$, and up to biholomorfism $X$ admits two antiholomorphic involutions with nonempty real part; the resulting real surfaces are the hyperboloid, with the real part $R X$ homeomorphic to torus, and the ellipsoid, with $R X \cong S^{2}$.

Fix a pair $P_{1}, P_{2}$ of generatrices of $X$. The fundamental classes $\left[C P_{1}\right],\left[C P_{2}\right]$ form a basis of $H_{2}(C X) \cong Z \oplus Z$. For any curve $A \subset X$ one has $[C A]=$ $m_{1}\left[C P_{1}\right]+m_{2}\left[C P_{2}\right]$ for some nonnegative integers $m_{1}, m_{2}$. The pair $\left(m_{1}, m_{2}\right)$ is called the bidegree of $A$. If $\left[x_{0}: x_{1}\right],\left[y_{0}: y_{1}\right]$ are homogeneous coordinates in $P_{1}$, $P_{2}$ respectively, $A$ is given by a polynomial

$$
F\left(x_{0}, x_{1} ; y_{0}, y_{1}\right)=\sum_{i, j=1}^{m_{1}, m_{2}} a_{i j} x_{1}^{i} x_{0}^{m_{1}-i} y_{1}^{j} y_{0}^{m_{2}-j},
$$

which is homogeneous of degrees $m_{1}$ and $m_{2}$ in $x_{0}, x_{1}$ and $y_{0}, y_{1}$, respectively.

In the case of hyperboloid the antiholomorphic involution conj acts by conjugating all the four coordinates; hence $A$ is real iff all $a_{i j}$ are real. In the case of 
ellipsoid conj acts via $\left(\left[x_{0}: x_{1}\right],\left[y_{0}: y_{1}\right]\right) \mapsto\left(\left[\bar{y}_{0}: \bar{y}_{1}\right],\left[\bar{x}_{0}: \bar{x}_{1}\right]\right)$, and $A$ is real iff $a_{i j}=\bar{a}_{j i}$ (in particular, $m_{1}=m_{2}$ ).

In order to encode the topology of a real curve on a quadric we use a modification of the standard encoding scheme used for plane projective curves, see, e.g., [10]. First, let $(X, c o n j)$ be a hyperboloid and $A \in X$ be a nonsingular real curve. The real part $R A$ may have components of two types: those contractible in $R X$ and those noncontractible; they are called ovals and nonovals, respectively. The number of ovals (of nonovals) is denoted by $l$ (by $h$ ). Each oval bounds a topological disk in $R X$, which is called the interior of this oval. The fundamental classes $\left[R P_{1}\right]$ and $\left[R P_{2}\right]$, endowed with some orientations (which are to be fixed), form a basis of $H_{1}(R X) \cong Z \oplus Z$. Let $N_{1}, \ldots, N_{h}$ be the nonovals of $A$. All of them realize the same nontrivial class $\left(c_{1}, c_{2}\right)$ in $H_{1}(R X)$, with $c_{1}, c_{2}$ relatively prime. So the real scheme of $R A \subset R X$ can be encoded by

$$
\left\langle\left(c_{1}, c_{2}\right), \text { scheme }_{1},\left(c_{1}, c_{2}\right), \text { scheme }_{2}, \ldots,\left(c_{1}, c_{2}\right), \text { scheme }_{h}\right\rangle,
$$

where scheme $_{1}, \ldots$, scheme $_{h}$ are the schemes of ovals in the connected components of $R X \backslash\left(N_{1} \cup \ldots \cup N_{h}\right)$, cf. [10], [8].

If $(X, c o n j)$ is an ellipsoid, all the components of $R A$ are ovals; their number is denoted by $l$. In this case we fix a point $\infty \in R X \backslash R A$, which is called the exterior point, and for an oval $C \subset R X$ define its interior to be the component of $R X \backslash C$ that does not contain $\infty$. This gives rise to a natural partial order on the set of ovals, and the scheme of ovals can be encoded as in [10].

Following F.Klein, see [11] or [1], we say that a real curve $A$ is of type I or type II if $R A$ divides or does not divide $C A$. If $A$ is of type I, the natural orientations of the components $U$ and $V$ of $C A \backslash R A$ induce a pair of opposite orientations on $R A=\partial U=\partial V$ called the complex orientations of $R A$. The real scheme endowed with the type and, in the case of the type I, with the complex orientations is the complex scheme of $A$. The type of a curve with a real scheme $\langle B\rangle$ is encoded via $\langle B\rangle_{I}$ or $\langle B\rangle_{I I}$.

All real curves of a given bidegree $(m, n)$ form a space $C_{m, n} \cong R P^{N}, N=$ $m n+m+n$. The set $\Delta \subset C_{m, n}$ of singular curves has dimension $N-1$. Denote by $S \subset \Delta$ the subset of curves that have a singular point other than a node or a cusp, or have several singular points. Then $\Delta \backslash S$ is a manifold (although not a smooth submanifold of $C_{m, n}$ ). The rigid isotopy class of a curve $A \in C_{m, n} \backslash \Delta$ (or $A \in \Delta \backslash S$ ) is the component of $C_{m, n} \backslash \Delta$ (respectively, $\Delta \backslash S$ ) containing $A$. The components of $C_{m, n} \backslash \Delta$ (or $\Delta \backslash S$ ) are chambers (respectively, walls).

3. Curves with a node or a cusp. First we enumerate the walls in $C_{3,3}$.

THEOREM 1. The number of walls in $C_{3,3}$ equals 20 in the case of hyperboloid and 6 in the case of ellipsoid.

Proof. Consider a curve $A \in \Delta \backslash S$, blow up its singular point, and blow down the two generatrices through this point. The result is a nonsingular quartic 
$Q \subset P^{2}$. The inverse transformation is given by a pair $\left(q_{1}, q_{2}\right)$ of distinct points in $Q$ (the images of the generatrices), which are real in the case of hyperboloid or complex conjugate in the case of ellipsoid. In the case of hyperboloid these points are ordered and the real line through them is oriented. If $A$ has a cusp, the line is tangent to $Q$.

Thus in the case of ellipsoid it is clear that the walls are enumerated by the rigid isotopy classes of real quartics; due to [11] their number equals 6 .

In the case of hyperboloid the walls are enumerated by the connected components of the space Conf of configurations $\left(Q, q_{1}, q_{2}, \epsilon\right)$, where $\epsilon$ is an orientation of the real line through $q_{1}$ and $q_{2}$. Thus in addition to the rigid isotopy type of $Q$ one needs to distinguish whether $q_{1}$ and $q_{2}$ belong to the same or distinct ovals of $R Q$. In the latter case denote the configuration $\left(Q, q_{1}, q_{2}, \epsilon\right)$ by $b_{\alpha}, \alpha=2,3,4$, if the real scheme of $Q$ is $\langle\alpha\rangle$, and by $b_{\text {inn }}$ (or $b_{\text {out }}$ ) if it is a nest $\langle 1\langle 1\rangle\rangle$ and $q_{1}$ lies in the inner (or outer) oval of the nest. (Here $\langle\alpha\rangle$ denotes $\alpha$ ovals lying outside of each other, and $\langle 1\langle 1\rangle\rangle$ denotes 2 ovals, one inside another.) In the former case denote the configuration by $a_{\alpha}, \alpha=1, \ldots, 4, a_{\text {inn }}$ (or $a_{\text {out }}$ ), respectively.

Besides, all the configurations except $b_{2}, b_{3}$ can be given a sign + or - in the following way. The points $q_{1}$ and $q_{2}$ divide the real line through them into two segments oriented via $\epsilon$; let $\overline{q_{1} q_{2}}$ be the one having $q_{1}$ as the origin. Denote the configuration $\left(Q, q_{1}, q_{2}, \epsilon\right)$ by $a_{\alpha}^{-}$(or $\left.b_{\text {inn }}^{-}, b_{\text {out }}^{-}\right)$if a neighborhood of $q_{1}$ in $\overline{q_{1} q_{2}}$ lies inside the oval containing the points $q_{1}$ and $q_{2}$ (or inside the outer oval of the nest), and by $a_{\alpha}^{+}$(or $b_{i n n}^{+}, b_{\text {out }}^{+}$) otherwise. For $b_{4}$ consider a one-sided topological circle $\gamma$ in $R P^{2}$ not intersecting $R Q \cup \overline{q_{1} q_{2}}$ and denote the configuration by $b_{4}^{-}$(or $b_{4}^{+}$) if the complex orientations of the two ovals containing $q_{1}, q_{2}$ are (respectively, are not) coherent in $R P^{2} \backslash \gamma$.

To complete the proof it remains to notice that the set of connected components of Conf is in a natural one-to-one correspondence with the set $\left\{a_{\alpha}^{ \pm}(\alpha=\right.$ $\left.1, \ldots, 4), a_{\text {inn }}^{ \pm}, a_{\text {out }}^{ \pm}, b_{2}, b_{3}, b_{4}^{ \pm}, b_{\text {inn }}^{ \pm}, b_{\text {out }}^{ \pm}\right\}$of 20 elements. The assertion is an obvious consequence of the following lemma.

Lemma on Permutation of Ovals. For any nestless real quartic $Q$ there is a rigid isotopy that takes $Q$ to itself and induces any given permutation of the ovals of $Q$. Besides, if $Q$ consists of two or three ovals, there is a rigid isotopy which takes every oval to itself and reverses the orientation of a given line crossing two of the ovals.

Proof. Since the rigid isotopy class of a real quartic is determined by its real scheme (see [11] or [1]) and since $P G L(3 ; R)$ is connected it suffices to realize each real scheme $\langle\alpha\rangle, \alpha=2,3,4$, by a quartic $Q$ and to find projective transformations of $Q$ inducing necessary rigid isotopies. If $\alpha=2$, one can take for $Q$ the curve $\left(4 x_{1}^{2}+4 x_{2}^{2}-x_{0}^{2}\right)\left(4 x_{0}^{2}+4 x_{2}^{2}-x_{1}^{2}\right)=t\left(x_{0}^{4}+x_{1}^{4}+x_{2}^{4}\right)$, where $t \in R$ is small. Then the transformation $\left(x_{0}, x_{1}, x_{2}\right) \rightarrow\left(x_{1},-x_{0}, x_{2}\right)$ permutes the ovals of $Q$. For $\alpha=3$ and 4 consider the cube $\left\{\left(x_{0}, x_{1}, x_{2}\right)|| x_{i} \mid \leq 1\right\}$ in $R^{3}$. There are four planes through the origin which intersect the cube in regular hexagons. Let 


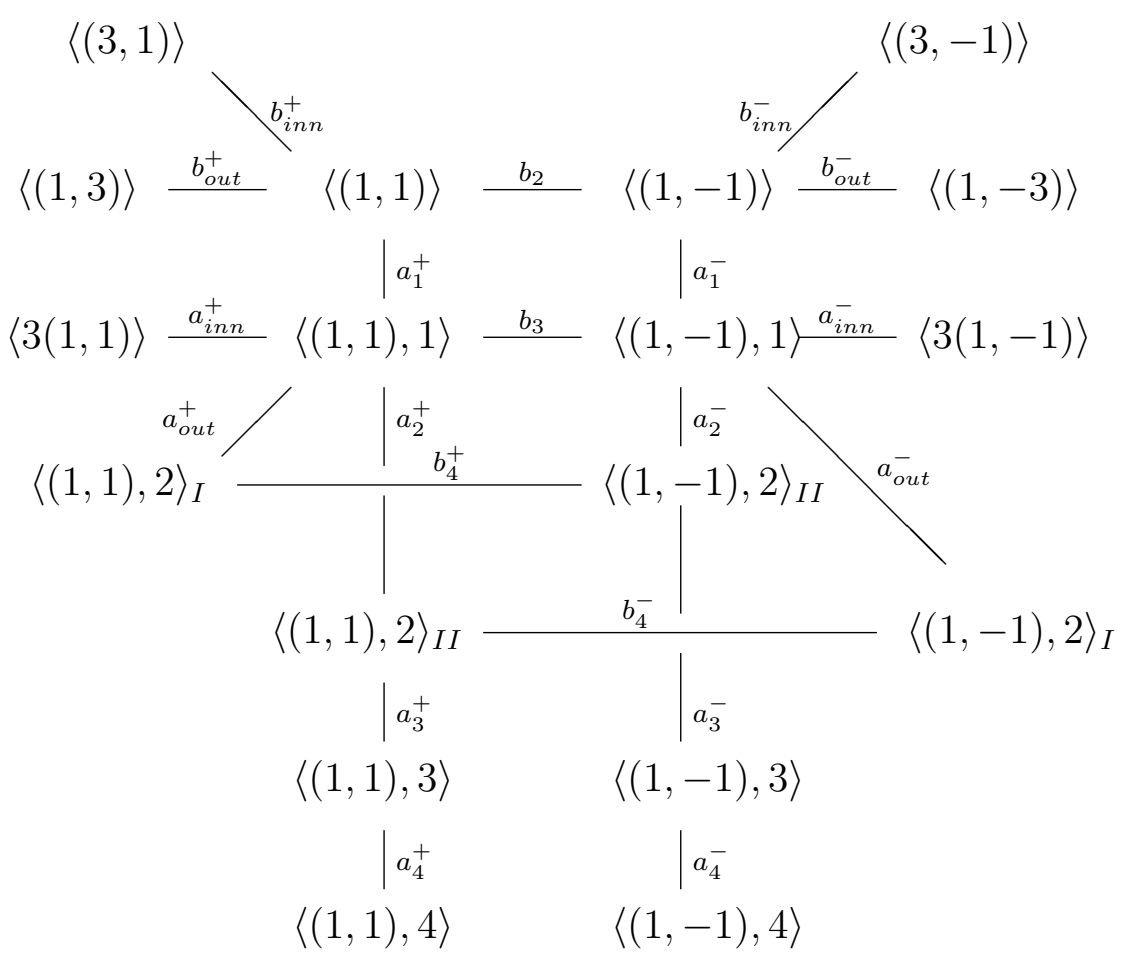

Figure 1: Chambers of curves of bidegree $(3,3)$ on a hyperboloid

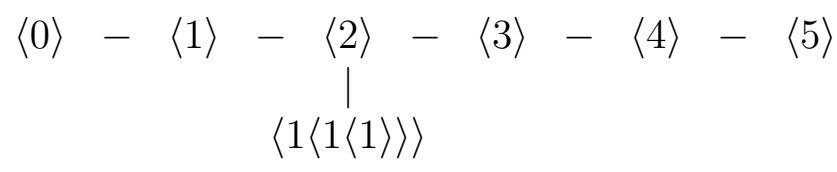

Figure 2: Chambers of curves of bidegree $(3,3)$ on an ellipsoid

$u\left(x_{0}, x_{1}, x_{2}\right)=0$ be the equation of the union of the planes. Then the equation $u\left(x_{0}, x_{1}, x_{2}\right)+t\left(x_{0}^{4}+x_{1}^{4}+x_{2}^{4}\right)=0$ with small $t \in R$ defines a nonsingular quartic $Q \subset P^{2}$, which, depending on the sign of $t$, has 4 or 3 ovals; they correspond to pairs of opposite vertices or, respectively, faces of the cube and, hence, any permutation of the ovals can be realized by a symmetry of the cube. Besides, for $\alpha=2$ and 3 the transformation $\left(x_{0}, x_{1}, x_{2}\right) \rightarrow\left(x_{1},-x_{0}, x_{2}\right)$ takes every oval to itself and reverses the orientation of the line $x_{2}=0$.

\section{Main result.}

THEOREM 2. The rigid isotopy classification of nonsingular real curves of bidegree $(3,3)$ on a hyperboloid (on an ellipsoid) coincides with the classification of their complex (real) schemes. The adjacency graphs of the chambers in $C_{3,3}$ are shown in Figures 1 (for the hyperboloid) and 2 (for the ellipsoid). 
ProOF.

Consider the exact sequence of $\left(C_{3,3}, \Delta, S\right)$ (recall that $\operatorname{dim} C_{3,3}=15$ )

$$
0 \rightarrow H_{15}\left(C_{3,3}, S\right) \rightarrow H_{15}\left(C_{3,3}, \Delta\right) \rightarrow H_{14}(\Delta, S) \stackrel{i n}{\rightarrow} H_{14}\left(C_{3,3}, S\right)
$$

(Here and below all homology groups have $Z / 2$-coefficients). It is clear that the number $c$ of chambers equals $\operatorname{dim}_{Z / 2} H_{15}\left(C_{3,3}, \Delta\right)$ and the number $w$ of walls equals $\operatorname{dim}_{Z / 2} H_{14}(\Delta, S)$. Since $H_{15}\left(C_{3,3}, S\right)=H_{15}\left(C_{3,3}\right)=Z / 2$, from the exactness of (1) it follows that $c=1+w-\operatorname{codim}_{Z / 2}$ ker $i n$.

In the case of ellipsoid due to Theorem 1 one has $w=6$ and, hence, $w \leq 7$. On the other hand, perturbing the corresponding singular curves one obtains seven chambers, which differ by the real schemes, and the statement follows.

In the case of hyperboloid the number of complex schemes of curves of bidegree $(3,3)$ equals 18 (see [8], §3.10). ${ }^{1}$ Thus, $c \geq 18$. Since $w=20$, see Theorem 1 , in order to prove the opposite inequality it suffices to show that $\operatorname{codim}_{Z / 2}$ ker in $\geq 3$. Let $x_{j}$ be the coordinates of a class $x \in H_{14}(\Delta, S)$ with respect to the basis of $H_{14}(\Delta, S)$ formed by the classes $w_{j}$ realized by the walls. Then $\operatorname{codim}_{Z / 2}$ ker in is the number of independent equations in $x_{j}$ that determine ker $i n$, and it remains to find three such equations. Due to the Alexander-Pontryagin duality one has

$$
H_{14}\left(C_{3,3}, S\right) \cong H^{1}\left(C_{3,3} \backslash S\right)=H o m\left(H_{1}\left(C_{3,3} \backslash S\right), Z / 2\right) .
$$

Hence, $x \in$ ker in if and only if in $x \circ H_{1}\left(C_{3,3} \backslash S\right)=0$, and the required equations are obtained by multiplying the relation in $x=\Sigma x_{j}$ in $w_{j}$ by three linearly independent classes of $H_{1}\left(C_{3,3} \backslash S\right)$. The latter are represented by small circles about $S$ centered at the points corresponding to the three curves shown in Figure 3. (These curves can easily be constructed by perturbing unions of lines. Note that the curves $b$ and $c$ differ by their complex orientations.) Each circle intersects only four walls, transversally at one point each. So from (2) it follows that the classes realized by the circles are independent and the corresponding equations are nontrivial.

\footnotetext{
${ }^{1}$ The number given in [8] is 9 , but one should take into account that the complex schemes are studied in [8] up to permutation of the factors of $P^{1} \times P^{1}$.
} 


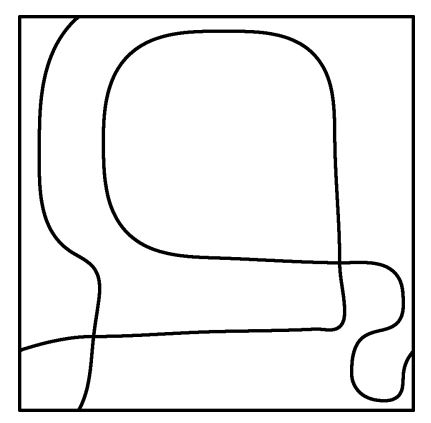

a

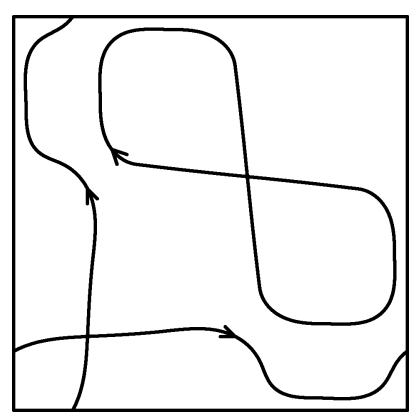

$\mathrm{b}$

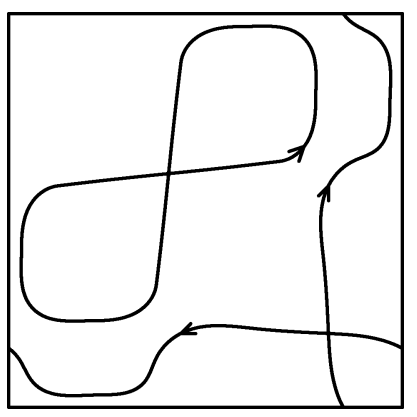

$\mathrm{C}$

Figure 3:

\section{References}

[1] V.A.Rokhlin. Complex topological characteristics of real algebraic curves // Uspekhi Mat. Nauk, vol.33, N.5, 1978, p.77-89. (Russian). English transl. in: Russian Math. Surveys, vol.33, 1978.

[2] V.M.Kharlamov. Rigid isotopic classification of real planar curves of degree 5// Funktsional. Anal. i Prilozhen., vol.15, N.3, 1981, p.88-89. (Russian) English transl. in: Functional Anal. Appl.,vol.16, N.3, 1981, p.88-89.

[3] V. V. Nikulin. Integral symmetric bilinear forms and some of their applications// Izv. Akad. Nauk SSSR, vol.43, 1979, N.1, p.117-177. (Russian). English transl. in Math. USSR-Izv., vol.14, 1980, p.103-167.

[4] A.I.Degtyarev. Appendix in: V.M.Kharlamov, V.A.Rokhlin, and O.Ya. Viro. Topological properties of real plane projective algebraic curves // to appear.

[5] V.I.Zvonilov. Stratified spaces of real algebraic curves of bidegree $(\mathrm{m}, 1)$ and $(\mathrm{m}, 2)$ on a hyperboloid// Amer. Math. Soc. Transl. (2), 1996, vol.173, p.253-264.

[6] D. A. Gudkov, A. K. Usachev. Nonsingular curves of small orders on a hyperboloid // in: Methods of the Qualitative Theory of Differential Equations, editor E. A. Leontovich-Andronova, Gor'kov. Gos. Univ., Gorki, 1980, p.96-103. (Russian).

[7] D.Hilbert. Ueber die reelen Zuge algebraischen Curven// Math. Ann., 1891, B.38, S.115-138.

[8] V. I. Zvonilov. Complex topological invariants of real algebraic curves on a hyperboloid and on an ellipsoid // Algebra i Analiz, vol.3, N.5, 1991, p. 
88-108. (Russian). English transl. in: St. Petersburg Math. J., vol.3, N.5, 1992, p.1023-1042.

[9] V.M.Kharlamov, V.A.Rokhlin, O.Ya. Viro. Topological properties of real plane projective algebraic curves // to appear.

[10] O.Ya.Viro. Progress in the topology of real algebraic varieties over the last six years // Uspekhi Mat. Nauk, vol.41, N.3, 1986, p.45-67. (Russian). English transl. in: Russian Math. Surveys, vol.41, 1986.

[11] F.Klein. Gesammelte mathematische Abhandlungen. B.2// Berlin, 1922; reprint, 1973.

Bilkent University, Ankara, Turkey

degt @ fen.bilkent.edu.tr, degt@pdmi.ras.ru

Syktyvkar University, Syktyvkar, Russia

zvonilov @ ssu.komi.com 